\title{
Sensor Triggered Replacement of Spare Parts: Customer Service Process Innovation
}

\author{
Muztoba Ahmad Khan ${ }^{1}$, Gabriela Lais Rozati ${ }^{1,2}$, Thorsten Wuest ${ }^{1}$ \\ ${ }^{1}$ West Virginia University, Morgantown, WV, USA \{mdkhan, \\ garozati\}@mix.wvu.edu; thwuest@mail.wvu. edu \\ ${ }^{2}$ School of Engineering of Piracicaba, Piracicaba, Brazil
}

\begin{abstract}
Customer services hold an increasingly dynamic and crucial role in today's highly competitive world. It is also one of the important factors that enable companies to sustain their competitive advantage. The purpose of this study is to develop a basic model of customer service process automation, which will support the spare parts replacement procedure. The idea is to use sensors for identifying the condition of spare parts in real time and initiate an automated replacement process at the right time. A conceptual model of an integrated customer service process automation, derived from a case study, is illustrated, which emphasizes the significance of information exchange between business processes and communication between supply chain collaborators. The suggested changes for the model aim to improve the service level, machine and part life, design of the part and also to reduce human errors during ordering process, overall cost and inventory level.
\end{abstract}

Keywords: Supply chain - Sensor - Intelligent products · Service automation · Internet of things $\cdot$ Predictive maintenance $\cdot$ Spare parts replacement

\section{Introduction}

To thrive in today's highly competitive world, business organizations must constantly look for innovative strategies to improve their competitiveness. The advancement of Information and Communication Technology (ICT) and its creative use in business has compelled industries to revolutionize their business proposition and the way they operate [1]. Competition also has driven industries to transform into customer-focused, service-oriented organizations, irrespective of the products and services they offer. Even manufacturing firms need to be aware of the service aspects because the service component of the product-service mix, e.g. Industrial Product Service Systems, offers the best opportunity of achieving sustainable competitive advantage and revenue streams along the whole product and/or service lifecycle [2].

Among different processes in an organization, the customer service process, such as availability and supply of spare parts, after-sales service or repair service on products,

adfa, p. 1, 2011.

(C) Springer-Verlag Berlin Heidelberg 2011 
affects customer satisfaction about products and purchases to a great extent [3]. The essence of today's business requires that firms interact with their customers and business partners using technology to provide these services instantaneously and globally. To do so, businesses can foster innovation to improve their service performance and efficiency that will ideally benefit both business and customer [4].

The aim of this paper is to (i) analyze the spare part replacement process within a company case study, (ii) identify the current shortcomings and issues and (iii) develop an innovative model of spare parts replacement process that will help to address the identified shortcomings and issues. The main idea is to use sensors to identify the current condition of spare parts and initiate an automated replacement process considering the lead time, inventory level, etc. The suggested changes for the model aim to improve the service level, machine and part life, design of the part and also to reduce human errors during ordering process, overall cost and inventory level.

This paper is organized as follows: Section 2 discusses the states of the art of sensor based predictive maintenance and business process reengineering. In Section 3, we first describe and then analyze the problems of the current spare parts replacement process within the boundary of our case study and then we propose our new customer service model. Section 4 discusses and concludes this paper.

\section{State of the Art}

In recent years, the utilization of sensors combined with condition monitoring is becoming more popular in many industries. Its application can be found in a variety of areas, e.g., on rotating equipment in order to measure vibration [10]; on military vehicles, mortar systems and other weapons to monitor wear-and-tear [8]; on bridges to record vibration, humidity, strain, inclination, load, cracks, etc. [7]; on diesel engines to verify the permittivity and viscosity of the fluids [9], on cars to measure tire pressure [6], [12], etc.

Sensor technology is rapidly improving and the availability of low-cost sensor is continuously growing. This enables applications and application scenarios that were not feasible until today. Condition-based monitoring, using sensors, algorithms and wireless communication, allows to identify when a part or equipment is in the process of failing and subsequently allows to take appropriate measures, e.g., repair, prior to the failure. This prevents machine downtime due to unexpected failure.

Commonly employed Preventive Maintenance (PM) schedules repairs, lubrication, adjustments, and machine rebuilds for all critical plant machinery at regular intervals, regardless of the performance of the equipment or machine. The main difference to Predictive Maintenance (PrM) is that the maintenance activities are performed at the time they are required. Based on findings from the US Department of Energy, the implementation of a functional predictive maintenance programs in the oil and gas industry has been shown, on average, 25 to $30 \%$ reduction in maintenance cost [5].

The application in the focus of this publication is the sensor based monitoring of spare parts to obtain information on current condition and identify potential issues and replacement needs at an early stage. Based on the sensor data, it is possible to predict 
the equipment's Residual Useful Life (RUL). The application of PrM can bring gains to all stakeholders of the supply chain, such as providing accurate lead time and preparing and executing the repair or the replacement of the part [11]. The focus of this research is not on the technical implementation of sensor-based monitoring but the combination of PrM with customer service processes and business process reengineering (BPR) of those to improve efficiency.

\section{Case Study}

In this section, a case study is presented to motivate the development of a new spare part replacement process. First, the case study and its background are illustrated before the current spare part replacement process, currently in use, is analyzed. Based on this analysis, existing issues of the process are identified and elaborated. Following, a new process is developed, aimed to address the previously identified issues.

\subsection{Background of Case Study}

The studied company is a manufacturer and service provider of construction equipment with a long history of manufacturing capital goods involving state of the art technology. This company will be referred to in the following as the Construction Equipment Manufacturer (CEM). The CEM operates globally with subsidiaries in all major markets while being headquartered in the United States. The CEM has been offering spare parts and technical field service for its equipment for a long time and has gradually added more services to its total offering. They pride themselves of superior customer service, making sure their customers will receive the best possible support to ensure their operations to run smoothly. This allowed them to build a reputation of high quality products and services, which in turn justifies their premium prices. CEM's customers include construction, agriculture, resource, energy and transportation industries. Thousands of small, medium and large organizations from these industries around the world utilize CEM's products and services.

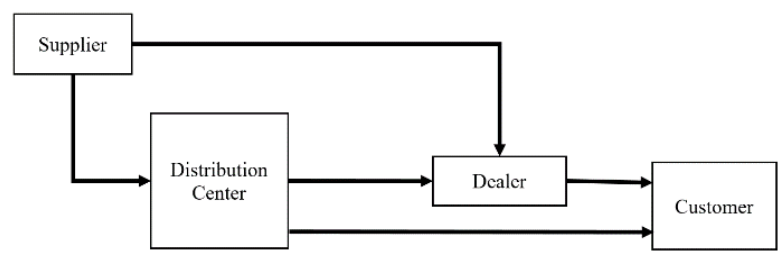

Fig. 1. CEM's simplified spare parts distribution chain.

The CEM currently offers thousands of spare parts for its equipment. In this study, the focus is on frequently replaceable parts that require minimal technical knowledge to replace (i.e. parts that can be easily diagnosed and replaced by end users). This is because a part that needs in depth technical knowledge to being serviced or repaired, 
will most likely require a different customer service process. Therefore, in order to being able to generalize the findings of the study, the scope is limited. Some of the most frequently replaced parts offered by CEM include, air filters, oil filters, batteries, radiators etc. Fig. 1 presents the company's simplified spare parts distribution chain. We see that these subsystems are typical for a classic distribution chain and hence the case which is presented here can be understood as being generalizable to some extent.

\subsection{Analyzing the spare part replacement process}

In this sub-section, the current spare part replacement process employed by the CEM is analyzed in detail with the goal of identifying potential issues and potential for improvement. The current spare part replacement process is initiated by customer demand. It can be triggered in different ways; for example periodic replacement, sensor based recommendation, failure of the machine etc. Next, the customer communicates his/her order to the CEM authorized dealer (e.g., via email or phone). The placed order arrives at the dealer with the following attributes: customer information, part identity number, required quantity, due date of the order and customer tolerance for tardy job. The customer tolerance for tardy job translates into different priority levels defined by the CEM; for example, priority level 1 indicates that the due date must definitely be met with no room for delays. If the inventory level at the dealer is sufficient to fulfill the order, the parts are sent to the customer from the dealer's inventory. Otherwise, the dealer reorders the required parts from the distribution center (DC). Then the DC determines a shipping date based on the order attributes, inventory level and lead-time. The DC may ship the part from its own facility or may ask the supplier to send it to the dealer. Based on feasibility, the DC may decide to send the parts directly to the customer, avoiding shipping them to dealer's location first. Fig. 2 illustrates the current spare part replacement process.

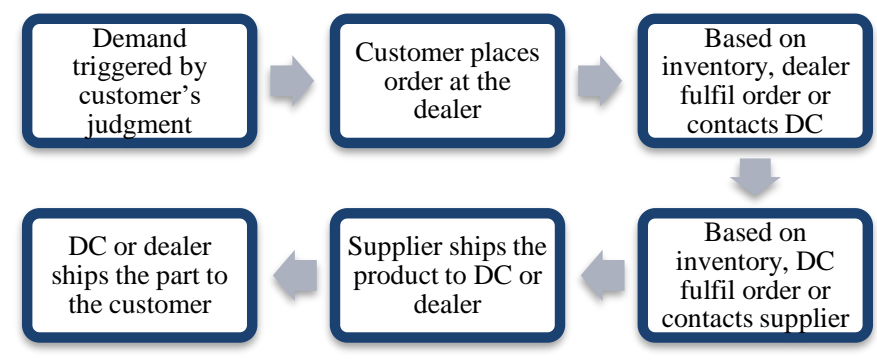

Fig. 2. Current spare part replacement process

\subsection{Shortcomings and issues of existing service process}

The current practice of the customer service process for frequently replaceable parts is successfully providing value to the customers. However, there is still potential for improvement and certain issues that may be addressed by a process redesign. Some of the challenges were indicated by the CEM personnel themselves as they previously 
emerged during operation. Other issues were identified in a comparative analysis. The identified issues are labeled as: suboptimal part and machine life, human errors during the process (especially critical was the ordering process), higher shipping cost and higher inventory level. Next, each category is explained separately, although some of them are interconnected.

Suboptimal part and machine life: The replacement process starts with customer demand, which is often triggered by the customer's judgment of the condition of that part. For some parts they depend on periodic replacement. But here, any kind of misjudgment by the customer can result in too early or too late replacement of the part. For example, air filters can sustain more than its average lifespan if it works in a clean environment compared to a dusty environment. If the filter gets replaced earlier than it is supposed to, we lose the span of the RUL of the filter. Fig. 3 illustrates this phenomenon. This is a waste of resources both environmentally and monetarily. If the part is overused (beyond its capability to fulfill its intended function), it may jeopardize the performance of the machine or even put other expensive parts or even the machine itself at risk.

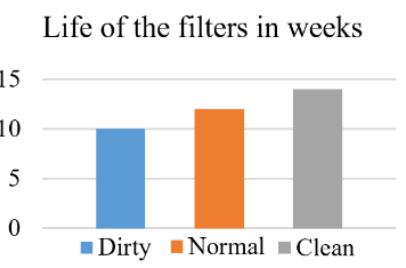

Fig. 3. Lifespan of air filters in different environments

Human errors during ordering process: As most of the orders are communicated manually through emails or phone calls, there is always a risk of human errors involved. For example, the current ordering process requires the customer to provide a unique part number to the dealer. Any mistake by the customer or dealer in communicating this number correctly may result in a faulty shipment or even the production of a wrong part.

Higher shipping cost: Sometimes customers wait until the machine breaks down, before ordering a required spare part; this is because they do not have sufficient information about the current condition of the parts. As a consequence, to minimize the idle time of the machine, customers ask the dealer to provide the spare parts within the shortest possible time. This kind of rush translates often directly into higher shipping cost. Whether these higher shipping cost are covered by the supplier or the customer, they are still an unnecessary waste of resources.

Higher inventory cost: Currently the dealers need to maintain relatively high inventory levels in order to being able to react to the volatile customer demand. This is because they do not have any information about the current condition of machines used by their customers. Maintaining high inventory levels is very costly as it not only binds capital through the stored material but also as it increases the requirements towards the storage facility and workers. Besides, some of the parts may start degenerating if they are kept waiting on the shelf for a long period of time and/or under certain conditions. 
Besides these four main issues presented, there are several other ones that can potentially benefit from a redesign of the process. Those include for example part design. With the implied access to real-life usage data within the new process, the part design can be improved based on a cross reference of the condition and cross-referenced environmental data. Leading to an improved design of parts for certain conditions, like application in desert (Hot, dry and dusty) environments.

\subsection{Proposed customer service model}

In this sub-section, the previously identified issues are taken as a basis for a reengineering of the current business process. Creating an new customer service model is initiated by the need to address the issues and challenges presented in the previous section. Because the future model handles process innovation at a conceptual and theoretical level, it is called the basic model of customer service process innovation.

The main idea of the proposed model adaptations is to automate the spare parts ordering process by means of real-time, sensor-based condition monitoring of spare parts. Sensor data along with customer data, such as inventory level, location, preferred shipping method and reordering quantity etc., will be stored in the cloud. Any of the stakeholders with proper authority will have access to this data in real-time. In the new model, customer demands will be triggered automatically based on the integration of customer and sensor data stored in the cloud in the supplier specific ordering systems. Depending on predetermined individual customer preference, the system may ask the customer to approve the order before processing or may automatically start processing the order at the DC. Real-time customer and sensor data will allow the system to find best possible solution in terms of cost, accuracy, lead time, shipping, inventory and quality without the additional effort of the currently employed system. Fig. 4 illustrates the new customer service process.

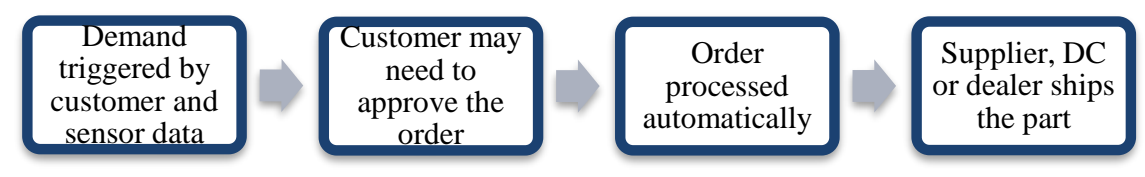

Fig. 4. New customer service process.

The proposed changes in the process will help customers to utilize the full RUL of a part. Additionally, it will prevent 'overuse' of a part beyond its RUL, which in turn will increase the overall machine life by avoiding failure during employment. The automated ordering process will also help to reduce potential human errors during the ordering and shipping process. The instantly available data will enable the system to plan a shipment before it will actually be needed at customer's inventory, which means that a slower but cheaper mode of transportation could be utilized while still meeting the customer demands on lead-time. When the CEM will have access to all of its customers' (relevant) data, it will be able to forecast demand more precisely, which in turn will 
help to reduce overall inventory across the whole supply chain (at both, the supplier and customer level). Better forecast will also allow to reduce the bullwhip effect.

The proposed model will be able to facilitate additional business-to-business (B2B) transactions such as invoicing and payment, reduction of procurement cost, and shortened lead-times better and more efficient than the traditional one. It may also have the potential to improve coordination and collaboration, both within and across companies due to the instantly available real-time data in the cloud. However, this has to be carefully assessed in further study and was not within the scope of this paper.

\section{Discussion and Conclusion}

After-sales customer services in manufacturing firms that are selling durable products have a strategic relevance in its potential contribution to a company's profitability, customer retention and product development [13]. Since customer service is a major source of competitive differentiation in today's business environment, many firms are trying to gain a competitive edge by innovating their customer service process. In this paper we have illustrated a case study of a leading Construction Equipment Manufacturer's spare part replacement process. We identified existing issues and scopes for improvement by analyzing the current process and proposed an improved conceptual model targeting these issues. The main idea of this proposed model is to automate the spare parts ordering process by means of real-time, sensor-based condition monitoring of spare parts making the data available in a cloud-based solution to all relevant stakeholders.

For small manufacturing firms this model may not be applicable because if the overall demand of spare parts remains relatively low, the proposed model might not be feasible from a financial perspective. However, some aspects, especially the direct connection to the customer's operation and access to use data might make it worthwhile.

The proposed model depends strongly on the reliability of the employed sensors. If a sensor fails to detect the current condition of spare parts with the required accuracy, then an incorrect order may be issued. However, this should not be an issue because sensor reliability is generally very high today.

Furthermore, the proposed model requires the customers to share information and data with the manufacturer. This might not be acceptable to some companies due to privacy issues. In this case it is important to ensure transparency of who has access and what data is exchanged for what application. Also there might be customers who may find this model too complicated for their operation and/or that want to remain in full control of their process and feel the automated variant is not providing that (felt) level of control.

In this paper we proposed a basic model of a customer service process innovation utilizing sensor technology and cloud infrastructure. The case study and the original spare part replacement process were analyzed and the process innovation was aimed to target the identified shortcomings. However, the proposed model only provides and outlook on the potential impact in full-scale industrial application. Further study needs to aim at operationalizing the proposed model and actually designing and implementing 
the technical solution, incl. sensors and cloud-connectivity to compliment the new business processes. Furthermore, additional use of the acquired data through the sensorbased condition monitoring is worthwhile to be explored. Potential areas are new services for Product Service Systems (PSS), innovations on the product design level and cross referenced analysis of the data for, e.g., environmental impact or worker safety solutions.

Acknowledgement. This work was partly funded by the Brazilian Coordination for the Improvement of Higher Education Personnel (CAPES) through the Science without Borders (CSF) fellowship program. The authors gratefully acknowledge the support of the CAPES. Finally, the authors would like to thank the reviewers for their helpful comments.

\section{References}

1. Rozendal, A., Lim, E. T., Tan, C. W.: A Change for the Better: Realizing BusinessIT Alignment Through Organizational Change. In 23rd European Conference on Information Systems (ECIS) (2015)

2. Gebauer, H., Kowalkowski, C.: "Customer-focused and service-focused orientation in organizational structures", Journal of Business \& Industrial Marketing, 27(7), 527-537 (2012)

3. Paparoidamis, N. G., Chumpitaz, R., Ford, J.: Service Quality, Customer Satisfaction, Value and Loyalty an Empirical Investigation in A Service Failure Context", Marketing Dynamism \& Sustainability. Springer, 173-173 (2015)

4. Visnjic, I., Wiengarten, F., Neely, A.: Only the Brave: Product Innovation, Service Business Model Innovation, and Their Impact on Performance", Journal of Product Innovation Management, 33(1), 36-52 (2016)

5. "Predictive maintenance: Is the timing right for predictive maintenance in the manufacturing sector?", Roland Berger. pp. 5-5 (2014)

6. Pohl, A., Ostermayer, G., Reindl, L., Seifert, F.: Monitoring the tire pressure at cars using passive SAW sensors. Ultrasonics Symposium, IEEE, 1, 471-474 (1997)

7. Casas, J. R., Cruz, P. J.: "Fiber optic sensors for bridge monitoring", Journal of Bridge Engineering, Vol. 8, Issue 6, pp. 362-373 (2003)

8. "U.S. Army Awards, http://www.thefreelibrary.com/U.S. Army Awards Augusta Systems \$1.18 Million Task Order to Monitor...-a0168777922

9. Scherer, M., Arndt, M., Bertrand, P., Jakoby, B.: Fluid Condition Monitoring Sensors for Diesel Engine Control", In Sensors, IEEE, 459-462 (2004)

10. Hashemian, H. M.: Wireless Sensors For Predictive Maintenance of Rotating Equipment in Research Reactors. Annals Nuclear Energy, 38(2), 665-680 (2011)

11. Compare, M., \& Zio, E.: "Predictive Maintenance by Risk Sensitive Particle Filtering", Reliability, IEEE Transactions, Vol. 63, Issue 1, pp. 134-143 (2014)

12. Brady, S., Van Order, D., Sharp, A.: "Advanced Sensors and Applications: Commercial Motor Vehicle Tire Pressure Monitoring and Maintenance", No. FMCSA-RRT-13-021, (2014)

13. Saccani, N., P. Johansson, and Marco Perona. Configuring the After-Sales Service Supply Chain: A Multiple Case Study. International Journal of Production Economics, Vol. 110, pp. 52-69 (2007) 